\title{
NUEVOS DESTINOS TURÍSTICOS EMERGENTES \\ Caso: Alto Valle Provincia de Río Negro - Patagonia Argentina
}

\author{
Ana Maria Boschi \\ Universidad del Comahue \\ Argentina. \\ amariaboschi@yahoo.com.ar \\ María Gabriela Torre \\ Universidad del Comahue \\ Argentina. \\ mgabytorre@yahoo.com.ar
}

\section{RESUMEN}

En la última década el turismo ha sumado nuevos lugares para la visita, como una opción complementaria de los destinos turísticos principales y del desarrollo local. El Alto Valle de la Provincia de Río Negro, Patagonia Argentina es uno de estos lugares definidos como "Destinos turísticos Emergentes" y objeto de políticas para el desarrollo turístico

Este artículo se centra en el análisis del Alto Valle de Río Negro como DTE, y su proceso de desarrollo con acento en la interacción entre los actores involucrados, las políticas y estrategias

Como resultado del análisis, se resalta que la política de inclusión al desarrollo turístico del Alto Valle de Río Negro, como un destino emergente, ha sido positiva ya que permitió canalizar una diversidad, complejidad y continuidad de acciones y compromisos de diferentes actores, que han promovido el surgimiento y consolidación de nuevos productos turísticos.

Palabras claves: Destinos Turísticos Emergentes 
THE NEW EMERGING TOURISM DESTINATIONS.

CASE STUDY FROM EL ALTO VALLE DE LA PROVINCIA DE RÍO NEGRO, PATAGONIA ARGENTINA.

\author{
Ana Maria Boschi \\ Universidad del Comahue \\ Argentina. \\ amariaboschi@yahoo.com.ar \\ María Gabriela Torre \\ Universidad del Comahue \\ Argentina. \\ mgabytorre@yahoo.com.ar
}

\begin{abstract}
In the last decade tourism has added new places to visit, as a complementary option of the main tourist destinations and local development. El Alto Valle de la Provincia de Río Negro, Patagonia Argentina is one of these places defined as "Emerging Tourism Destinations" (ETD) and this are object of policies for the development of tourism. This article focuses on the analysis of the Alto Valle del Río Negro as ETD, and its development process with emphasis on the interaction between the actors involved, policies and strategies. As a result of the analysis, it is emphasized that the policy of inclusion tourism development in El Alto Valle de la Provincia de Río Negro, as an Emerging Tourist Destination, has been positive in that it enabled a channeling diversity, complexity, and continuity of actions and commitments of different actors, which have promoted the emergence and consolidation of new tourist products.
\end{abstract}

Key words: Emerging Tourism Destinations. 


\section{INTRODUCCION}

En la última década el turismo ha sumado nuevos sitios, que poseen diversidad de atractivos potenciales, con una débil puesta en valor, teniendo por resultado un incipiente desarrollo turístico. Estos sitios son considerados "Destinos Turísticos Emergentes (DTE)".

Desde el Proyecto de Investigación denominado "Áreas Naturales, Áreas Naturales Protegidas y el Uso Público Turístico Recreativo Sustentable" Facultad e Turismo, de la Universidad del Comahue (2009) resulta de interés el análisis de estos espacios, dado que son nuevas áreas naturales que se incorporan al Turismo, impactando en la región.

Su desarrollo, desde una perspectiva endógena permite la "multiplicación de las acciones locales en la renovación de actividades tradicionales..., la apertura de nuevos canales comerciales, la revitalización de la pequeña empresa" (Arocena ,1995). En este sentido, los DTE promovidos, potencian a los pequeños y medianos emprendimientos, así como ponen en valor o en recuperación ciertas localidades del interior del país, teniendo como elemento dinamizador al turismo, el que genera nuevos espacios y oportunidades para su desarrollo. Albuquerque (2001) lo denomina "diversificación productiva como factor de solidez de la economía local", siguiendo la misma línea de lo que proponen Boscherini y Poma (2000) para el desarrollo de las economías locales.

El Alto Valle de Río Negro, localizado al norte de la Patagonia Argentina, es considerado un Destino Turístico Emergente -DTE- . Si bien tiene un alto potencial de atractivos naturales dado por la presencia del río que atraviesa de Oeste-Este al ambiente de áridas mesetas y una historia de poblamiento que reunió un crisol de razas y costumbres, el turismo no ha sido una política de desarrollo.

A partir del 2005, el Ministerio de Turismo de Río Negro inicia una etapa de estrategias conjuntas público- privadas, posicionando al Alto Valle como DTE, sobre la base de productos turísticos como la observación de aves, flotadas en el río, trekking en mesetas, y las Rutas del Vino, de los Colonos, del Riego, entre otros. 
Estas estrategias se orientan al desarrollo endógeno, facilitando iniciativas locales de desarrollo social, económico y ambiental turístico. De esta manera se promueve el "emponderamiento" de los recursos humanos locales en base a las necesidades del territorio, la facilitación de redes, y la participación del sector privado, en un proceso, que permite que lo "local" sea un espacio donde las iniciativas de los diversos actores de la sociedad organizada se vuelvan realidad (Vázquez Barquero, 2000), y donde "lo social se integra con lo económico" (Arocena, 1995).

El proceso de planificación, como una herramienta para la implementación del enfoque del desarrollo endógeno, facilita la definición de una estrategia más adaptada a los problemas, recursos e idiosincrasia de cada territorio" (Albuquerque, 1994). Ejemplo de ello son las Asociaciones Civiles Ruta del Vino, formada para su autogestión.

Asimismo, se observa también que si bien es importante considerar que las iniciativas de desarrollo local deben partir de sus - es decir los próximos al territorio Hay iniciativas que pueden resultar de mayor potencialidad si son coordinadas con los planes y estrategias de desarrollo regional o nacional, tal como es el caso de los planes: "Consolidación del Enoturismo para Argentina" del ministerio de Turismo de la nación en 2010 y "Plan estratégico de desarrollo turístico de las Rutas Alimentarias Río Negro" del ministerio de turismo de la provincia de Río Negro. En coincidencia con Madoery (2008), se puede observar que en estos programas, si bien el Estado asume el rol de impulsor del desarrollo local, se reconocen las diversidades territoriales, y el desarrollo está ligado principalmente al esfuerzo de ciudades y regiones que tratan de impulsar iniciativas y de transitar su propia experiencia.

Todas estas consideraciones se basan en las conexiones existentes entre Turismo y Desarrollo endógeno, aunque merecen especial tratamiento los eslabonamientos productivos o "clusters", la gestión y manejo de las fuentes de financiación y/o crédito, la promoción de inversiones, y la disponibilidad y/o acceso a los recursos tecnológicos. De todas maneras, el turismo entendido como una actividad diversificadora y dinamizadora del territorio, con base en los recursos de cada localidad, puede convertirse en una actividad económica complementaria, y/o principal, que impulse el proceso de desarrollo 
en los DTE- Destinos Turísticos Emergentes, tanto "desde la perspectiva de la eficiencia económica, del equilibrio ambiental, como de la equidad social" (Cotorruelo Menta, 2001).

En este sentido, el territorio posee otro valor por contener una cultura propia con sus ventajas comparativas (Wallingre, 2007 en Garbelotti et al 2009) tal el caso de ser la historia de productora mayor de manzanas y peras, y de poseer el río más importante de la Patagonia entre otros recursos potenciales.

En el análisis del DTE “Alto Valle de Río Negro" se parte del concepto de desarrollo que implica al "conjunto de capacidades generadas endógenamente, ligadas a la calidad de los recursos humanos, la capacidad organizativa y de articulación público-privada, la innovación y emprendidorialidad de los agentes locales, la capacidad institucional territorial, donde los impulsos exógenos se incorporan a un territorio organizado con una estructura de relaciones establecidas y consolidadas" (Madoery, 2001 b).

En este sentido, el programa de "Rutas Alimentarias" contiene el desarrollo de formas asociativas civiles, las cuales se han constituido para cada ruta (EJ Ruta del Vino), motorizando el desarrollo del DTE Alto Valle. Estas Asociaciones Civiles posibilitan mayor capacidad de autogobierno a las autoridades territoriales y refuerzan el despliegue de los valores socioculturales locales. De este modo se facilita la definición de una "estrategia de desarrollo endógeno más adaptada a los problemas, recursos e idiosincrasia de cada territorio" (Albuquerque, 1994).

Teniendo en cuenta estas concepciones, se analizan las principales características del Alto Valle como Destino Turístico Emergente (DTE), así como la interacción entre los actores involucrados, las políticas enunciadas y estrategias de desarrollo. El estudio se basa en la recolección de datos y en entrevistas semi-estructuradas cualitativas a informantes claves con un análisis comparativo e integral.

\section{Caracteristicas del Turismo en Alto Valle}

Esta región del Alto Valle de Río Negro, se localiza al norte de la Patagonia, 
y tiene desarrollo lineal a lo largo de $100 \mathrm{~km}$. aprox., siguiendo al río Negro. Sus características climáticas de temperaturas, sequedad, y amplitud térmica significativa (hasta $\operatorname{los} 20^{\circ}$ ), hacen de la fruticultura bajo riego, una actividad principal destacándose peras, manzanas y viñas, con intensos y brillantes colores en sus vinos. El "terroir" natural ofrece un hábitat ideal para varietales tintos como el merlot, el pinot noir y el malbec. Entre los blancos se destacan el semillón y el sauvignon ${ }^{1}$.

Los asentamientos en el Alto Valle tienen una disposición lineal a lo largo de su desarrollo, destacándose Cipolletti, Allen, Gral. Roca, Villa Regina, Cinco Saltos (Fig No 1 ).

Fig. 1: Asentamientos del DTE Alto Valle de Rio Negro

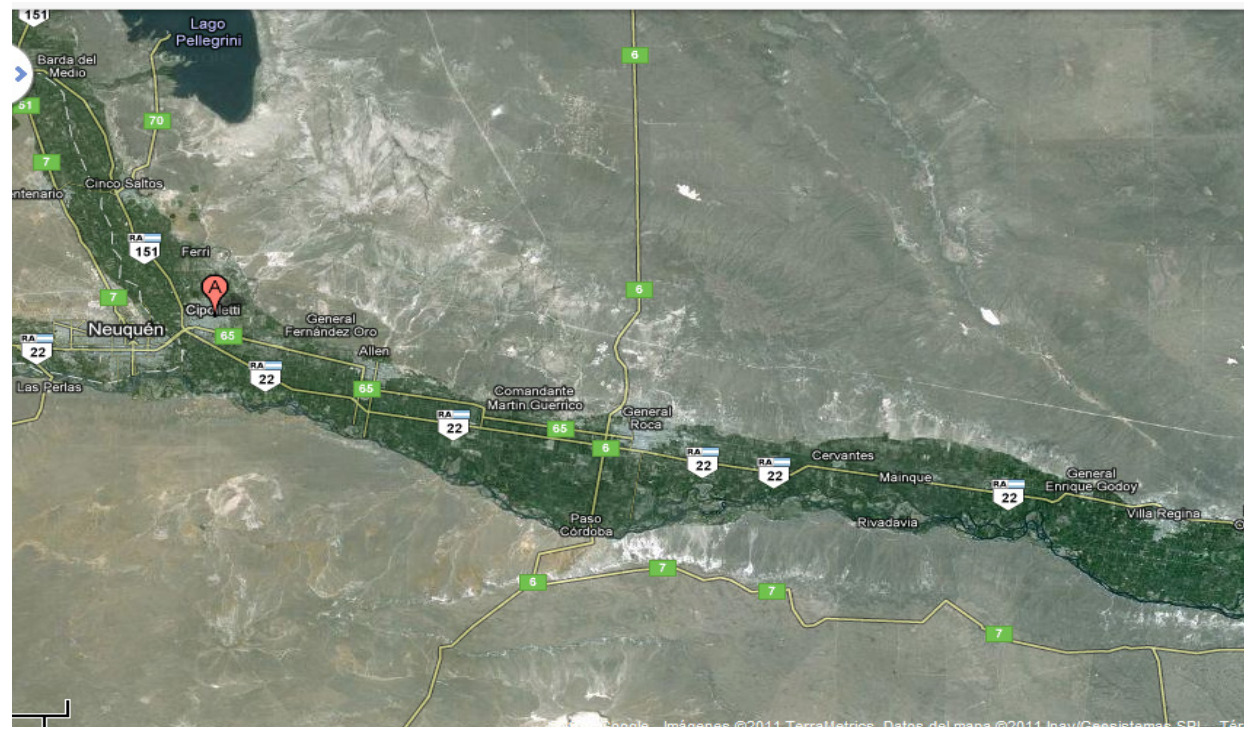

Fuente Elaboración propia en Base a Google Earth

El Alto Valle, forma parte de los programas institucionales de Provincia y Nación: "Plan de Desarrollo Turístico de Río Negro 2007-2015”, y "Plan Federal Estratégico de Desarrollo Turístico Sustentable (PFEDTS, 2016)", donde es tenido en cuenta como "DTE". En este contexto, el Ministerio de Turismo de Río Negro, ha iniciado un proceso

1 http://www.rionegrotur.gob.ar/index.php/zonas-turisticas/zona-valle/atractivos-turisticos/ruta-del-vino.html 
de desarrollo, aún en estadio inicial, con estrategias que priorizan el desarrollo de nuevos productos relacionados con los recursos de base de la región: el río Negro, obras de regadío de la región, el Lago Pellegrini, Mesetas-Bardas, paleontología asociada al ANP Valle Cretácico, infraestructura del Ferrocarril Gral. Roca, el zoológico Bubalcó entre lo más salientes. Promueve a prestadores locales especializados en productos turísticos regionales como: la "Ruta del Vino", "Flotadas en el río", "Observación de flora y fauna en cautiverio y al aire libre", "Trekking en las bardas", "pesca embarcada", "Ruta de las Manzanas y Peras", la "Ruta de los Pioneros", el Agroturismo, el Turismo Paleontológico y una serie de actividades recreativas que organizan los municipios para fechas especiales".

Cada producto turístico recreativo posee distinto nivel de complejidad y desarrollo, destacándose Ruta del Vino (Fig. 2) por su mayor consolidación e integración a nivel regional con la ruta del vino de Neuquén y con las demás provincias de la Argentina (www.caminosdelvino.org.ar).

Fig. 2 Ruta del Vino del Gran Valle en Patagonia

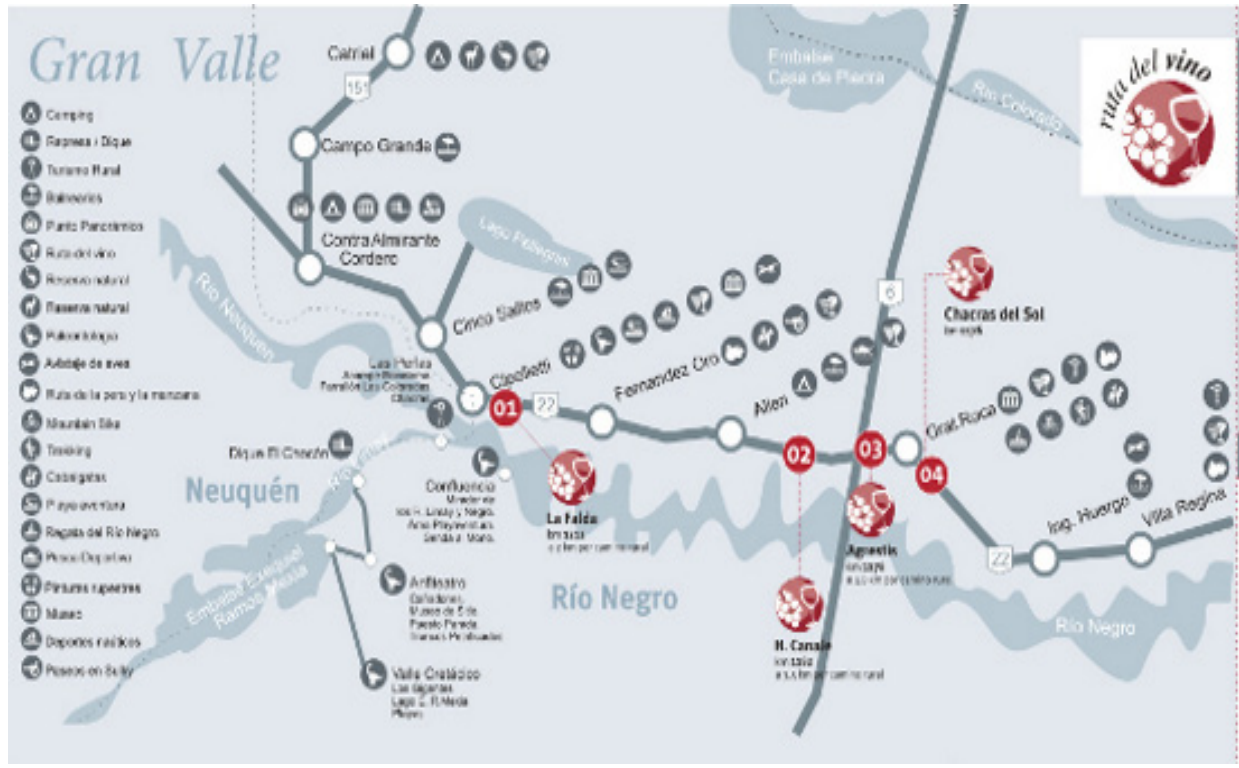

Fuente:http://www.patagonia.com.ar/General+Roca/454_La+ruta+del+vino+en+Alto+Valle.html

A partir de los avances en las políticas Turísticas (Plan Estratégico de 
Turismo 2007-2015) del Ministerio de Turismo de la Provincia de Río Negro, se inicia una tendencia a la incorporación de nuevos productos a través de la movilización de las inversiones de locales, con la prestación de servicios de agroturismo (casa de té, degustaciones especializadas, otras) y de otros atractivos como museos temáticos de colecciones particulares. Este crecimiento paulatino de la oferta turística recreativa del privado, fortalece, incrementa, mejora y hace sostenible las acciones, que desde el estado provincial con la coordinación de los gobiernos locales se han ido ejecutando (ej: inversión millonaria privada en el Zoológico Bubalcó).

En cuanto a la gestión Turística Recreativa, estas ciudades con un turismo incipiente en su organización, promueven el desarrollo turístico recreativo abordando variados perfiles de gestión: como facilitadores de actividades con un fuerte vínculo con el Estado (ej. Municipios del Alto Valle Este), dominancia en la planeación de la actividad (ej: Cipolletti), y con predomino en la organización y promoción de eventos locales y regionales (Ej: Cinco Saltos).

En tanto los Municipios antes mencionados, han tenido una baja participación, desde el año 2007 se han ido involucrando a partir de las políticas sectoriales que promovieron la creación de un área de gestión Turística Municipal. Es una excepción Gral. Roca que cuenta con el área de Turismo a partir de 1999. En el caso de las pequeñas localidades como Cervantes, Mainqué, Ing. Huergo, Godoy, y Chichinales se han agrupado para trabajar de manera coordinada por un profesional de Turismo, en la oferta de lo que denominaron ruta de los Pioneros.

Desde la Nación, a través del Plan Federal Estratégico de Desarrollo Turístico Sustentable (PFEDTS, 2016), que tiene como DTE al Alto Valle de la Provincia de Río Negro, a partir del 2010 se han desarrollado las primeras acciones de promoción nacional de los productos turísticos que fueran armados en el contexto público: ProvinciaMunicipios-Privados, e internacional para el producto Ruta del Vino. El Plan incorpora a los DTE asociados a destinos reconocidos, como política de captación y ampliación de la oferta turística con opciones complementarias, que están dirigidas a canalizar el cambio en el comportamiento, segmentación, estadía y actividades de los visitantes. 
En lo referido a excursiones, aún existe una débil presencia de los prestadores privados, por lo que aparece en competencia el Municipio promoviendo productos y actividades (por ej. General Roca organiza sus excursiones o el Ente para el Desarrollo de la Margen Sur - ENDEMAS.). Asimismo la Secretaría de Turismo Provincial, con sede en Cipolletti, constituye una base regional (desde la política provincial) que ha promocionado al privado en actividades regionales (pesca - flotadas) en donde al menos Empresa de Viajes y Turismo (Disp. Nac. 780/2011) La promoción de la región se realiza en Ferias nacionales, internacionales y regionales.

\section{Sentido de lo regional}

Las características de los destinos seleccionados se describen de forma sistémica, entendiendo la interacción que entre ellos se da, ya sea por el aprovechamiento de atractivos de manera no exclusiva, los prestadores que unifican más de un atractivo en un recorrido por el carácter de emergente de la región. De esta manera es una fortaleza la disposición geográfica del Valle, su linealidad en los asentamientos, la cercanía y la distribución de atractivos naturales y culturales que permiten dar significado integrador a la oferta turística

El DTE Alto Valle conforma un espacio turístico inicial, en donde Cipolletti, Cinco Saltos, Allen, Gral. Roca, Villa Regina, son centros de prestación de servicios varios: alojamiento, gastronomía, excursiones entre otros

En cuanto a la tipología de visitantes se distinguen en el caso del DTE Alto Valle son:

- Turistas: aquellos que no viven en las localidades del DTE y pernoctan en alguna de ellas. Son en un 80 \% de tipo corporativo, o familia de residentes, turistas de paso que al buscar información se quedan a conocer y otros solo aprovechan para conocer en vistas cortas. En el caso del corporativo se da por la presencia de empresas exportadoras de frutas que reciben empresarios que además de sus negocios buscan el placer, contratando servicios varios: "Catas y gastronomía en la Ruta del Vino", en Chacras, cabalgatas, flotadas...entre los más comunes.

- Recreacionistas - Excursionistas: aquellos que realizan excursiones y actividades recreativas diversas y viven en alguna de las localidades del DTE o zonas aledañas 
(como Neuquén ciudad por ej.) Suelen organizar previamente las salidas (ej excursiones de pesca y flotadas en el río negro). Entre ellas también se incluyen las vistas escolares programadas entre diversos municipios de la región

- Residentes: son aquellos habitantes de alguna de las localidades que realizan actividades recreativas en su lugar de origen. En este caso suelen organizar las actividades previamente, enterándose por medios de comunicación y difusión local (por ej. El habitante de Cinco Saltos que pasa la tarde en el lago Pellegrini). Entre ellos también incluyen las visitas escolares

Las principales ciudades del DTE Alto valle poseen oficinas de Informe de Turismo, la mayoría localizadas sobre el principal acceso, la ruta nacional $\mathrm{N}^{\circ} 22$. Esta decisión de localización está dada en función de la importante cantidad de turistas que pasan por esta ruta, en camino hacia la cordillera y de visitantes chilenos hacia el mar. En el período 2007 - 2011 esta estrategia ha sido muy positiva ya que aumentó significativamente el número de visitantes a emprendimientos que no tenían o poseían muy bajo registro de turistas (Ej; Bodega Agrestis, Chacra Blanquita Lainos, entre otros)

El registro de camas y visitantes en temporada Diciembre 2010 a Marzo 2011 y Semana Santa se presenta en la siguiente tabla.

Tabla 1 registro de visitantes en Oficinas de Turismo DTE Alto Valle

\begin{tabular}{|l|c|c|c|c|c|c|c|}
\hline & Camas & Temporada & $\begin{array}{c}\text { Semana } \\
\text { santa }\end{array}$ & $\begin{array}{c}\text { fines de } \\
\text { semana } \\
\text { largos }\end{array}$ & Recreacionistas & Turistas & Total \\
\hline Allen & $\mathbf{1 9 1}$ & 26 & 5 & 7 & $\mathbf{3 8}$ & 0 & $\mathbf{3 8}$ \\
\hline $\begin{array}{l}\text { Cinco } \\
\text { Saltos }\end{array}$ & $\mathbf{3 4}$ & $\mathbf{7 3 8 7 3}$ & $* * * * * *$ & & $* * * * * * *$ & $* * * * * *$ & 73873 \\
\hline Cipolletti & $\mathbf{6 9 7}$ & 46000 & 2000 & 3000 & 0 & $\mathbf{5 1 0 0 0}$ & $\mathbf{5 1 0 0 0}$ \\
\hline $\begin{array}{l}\text { General } \\
\text { Roca }\end{array}$ & $\mathbf{6 7 5}$ & 4048 & 673 & 801 & $\mathbf{5 0 0}$ & $\mathbf{5 0 2 2}$ & $\mathbf{5 5 2 2}$ \\
\hline TOTAL & $\mathbf{1 8 0 3}$ & $* *$ & $* *$ & $* *$ & $* *$ & $* *$ & $\mathbf{1 3 0 4 3 3}$ \\
\hline
\end{tabular}

Fuente: Ministerio de Turismo de Rio Negro (datos proporcionados por Áreas de Turismo Municipal y Prestadores de Servicios Diciembre-Marzo 2010-11

Los datos mencionados en la tabla 1 constituyen el registro realizado en las oficinas de Informes de las localidades del DTE. El mayor número de visitantes lo tiene 
Cinco Saltos, dado fundamentalmente por la afluencia de visitantes al lago Pellegrini al ser temporada estival. Asimismo Cipolletti en segundo lugar absorbe especialmente turistas en tránsito, General Roca y Allen se distinguen por el acento en los recreacionistas, contando con permanentes programas de actividades que pueden ser consultados en las oficinas locales En el caso de General Roca en particular la motivación para conocer la ciudad se debe a sus servicios.

En cuanto a la capacidad hotelera del DTE se registran un total de 1803 camas, siendo Cipolletti y General Roca los centros de mayor número de camas

En el Alto Valle, las ciudades mayores, cuentan con Oficinas de Informes Turístico localizadas sobre la principal ruta nacional 22, las cuales poseen funciones y vínculos interinstitucionales preestablecidos (tabla 2). La mayoría centra sus funciones en la Planificación y promoción de atractivos y actividades. Para el caso de General Roca se observa un mayor alcance en sus funciones dado por el carácter de ser Dirección de turismo y estar constituida con mayor antigüedad. Las oficinas, mantienen vínculos con otras instituciones gubernamentales, ONGs y privados. Están en relación a nivel de Estado con el Ministerio Turismo -Secretaría de Turismo de la Provincia de Rio Negro (sede Cipolletti), con los prestadores de turismo receptivo, diversos Entes u organizaciones de desarrollo como: ENDECIC (ente para el desarrollo del Lago Pellegrini, Cinco Saltos, Cte. cordero y Barda), AMRVC Asociación de Municipios de la Región de los Valles y la Confluencia IINTA (Instituto Nacional de Tecnología Agropecuaria), ENDEMAS Ente para el desarrollo de la Margen Sur, que interactúan ya sea en casos locales o regionales según el ámbito que abarquen. 
Tabla 2 Funciones y vínculos de las oficinas de turismo DTE Alto Valle

\begin{tabular}{|c|c|c|}
\hline Localidad & Funciones & Vinculos \\
\hline $\begin{array}{c}\text { Dirección } \\
\text { de Turismo } \\
\text { de General } \\
\text { Roca }\end{array}$ & $\begin{array}{l}\text { - Puesta en valor de los atractivos y de los } \\
\text { establecimientos productivos. } \\
\text { - Implementación de registro de consultas de } \\
\text { visitantes y análisis de estadísticas - } \\
\text { - Diseño y elaboración de material gráfico en } \\
\text { diferentes formatos. Y cartelería } \\
\text { - Implementación de excursiones educativas } \\
\text { gratuitas. } \\
\text { - Cursos de capacitación para público y el } \\
\text { rubro hotelero-gastronómico. } \\
\text { - Organización de eventos y participación de } \\
\text { diferentes eventos y ferias } \\
\text { - Incorporación de alojamiento extra hotelero. } \\
\text { - Promoción y difusión de la oferta turística y } \\
\text { recreativa }\end{array}$ & $\begin{array}{l}\text { - Instituciones Gubernamentales: } \\
\text { - Subsecretaría de Turismo de Río } \\
\text { Negro. Sede Cipolletti. } \\
\text { Privados: } \\
\text { Prestadores Turismo Rural: } \\
\text { Prestadores Turismo Aventura: } \\
\text { Establecimientos Hoteleros: } \\
\text { Alojamiento Extra-Hotelero }\end{array}$ \\
\hline $\begin{array}{c}\text { Oficina de } \\
\text { Turismo } \\
\text { Cinco Saltos }\end{array}$ & $\begin{array}{l}\text { - Planificación y desarrollo de nuevos productos, } \\
\text { actividades, } \\
\text { - Organización de eventos y festividades } \\
\text { turísticas, recreativas y culturales. } \\
\text { - Gestión de nuevos servicios y actividades } \\
\text { privadas. }\end{array}$ & $\begin{array}{l}\text { - Subsecretaría de turismo de Río } \\
\text { Negro. } \\
\text { - Ministerio de Turismo de la } \\
\text { provincia de Río Negro. } \\
\text { - ENDECIC (ente para el desarrollo } \\
\text { del L. Pellegrini, Cinco Saltos, Cte. } \\
\text { cordero y Barda } \\
\text { - Clubes como el de Leones, Leo, } \\
\text { Rotary, Rotaract e Interact, Náutico, } \\
\text { Cuerpo de guardavidas del Lago } \\
\text { Pellegrini, Fundación Luciérnaga, } \\
\text { Asociación }\end{array}$ \\
\hline $\begin{array}{c}\text { Oficina de } \\
\text { Turismo } \\
\text { Allen }\end{array}$ & $\begin{array}{l}\text { - Promoción de la ciudad: (Diseño de material } \\
\text { gráfico, asistencia a ferias, asistencia a Fiestas } \\
\text { Nacionales/Provinciales con la Reina de la } \\
\text { Pera) } \\
\text { - Comunicación de actividades a través de } \\
\text { medios gráficos, radiales y televisivos. } \\
\text { - Diseño y gestión de actividades recreativas } \\
\text { programadas por este área: } \\
\text { - Programación de Cursos de Capacitación } \\
\text { ligados al turismo. }\end{array}$ & $\begin{array}{l}\text { - Asociación de Municipios } \\
\text { (AMRVC) } \\
\text {-INTA (Instituto Nacional de } \\
\text { Tecnologìa Agropecuaria) } \\
\text { - Secretaria de Desarrollo Turístico. } \\
\text { Agencia Centro. } \\
\text {-Zoológico Bubalcó -Museo } \\
\text { Municipal } \\
\text {-Clubes de servicios comunitarios y } \\
\text { otros } \\
\text {-Hoteles -Prestador de servicios de } \\
\text { turismo aventura }\end{array}$ \\
\hline $\begin{array}{l}\text { Oficina de } \\
\text { Turismo } \\
\text { Cipolletti }\end{array}$ & $\begin{array}{l}\text { - Planificación de actividad turística y recreativa } \\
\text { en la ciudad } \\
\text { - Implementación de políticas de desarrollo } \\
\text { y fomento de la actividad: circuito histórico, } \\
\text { productivo (turismo rural), } \\
\text { - Organización de eventos } \\
\text { - Organización de Programas de Educación } \\
\text { Ambiental - Programa Anfitrión Rural }\end{array}$ & $\begin{array}{l}\text { - Prestadores Turísticos } \\
\text { - Escuelas primarias públicas y } \\
\text { privadas } \\
\text { Instituciones varias } \\
\text { - el Ministerio Turismo y Subsecretaría } \\
\text { de Turismo de la Provincia de Rio } \\
\text { Negro, Secretaría de Desarrollo } \\
\text { Turístico (Agencia zona centro de } \\
\text { Cipolletti) } \\
\text { - ENDEMAS }\end{array}$ \\
\hline
\end{tabular}




\begin{tabular}{|c|c|c|}
\hline $\begin{array}{c}\text { Turismo Alto } \\
\text { Valle Este }\end{array}$ & $\begin{array}{l}\text { - informar y derivar a los visitantes hacia los } \\
\text { emprendimientos nuevos de las localidades } \\
\text { que conforman la- ruta de los pioneros- son: } \\
\text { Huergo, Mainque, Chichinales, Cervantes y el } \\
\text { propio Godoy } \\
\text { - En Verano o sea temporada alta se desplaza la } \\
\text { parte de informes a Chichinales, local al lado } \\
\text { de la policía caminera .......- en la bajada de } \\
\text { Chichinales, cuando ingresas al valle- }\end{array}$ & $\begin{array}{l}\text { con las necesidades de los } 5 \\
\text { municipios ,, y los protestadores } \\
\text { que están en actividad, escuelas y } \\
\text { Ministerio del Turismo,-- Secretaria } \\
\text { de Turismo }\end{array}$ \\
\hline
\end{tabular}

Fuente: Elaboración propia en base a consultas con las Áreas de Turismo Municipal - 2011

En cuanto a los prestadores de turismo receptivo existe diversidad, por cuanto están aquellos que programan, venden y realizan las excursiones : flotadas en balsas por el Río Negro, con trecking, avistaje de aves - observación de restos fósiles, pesca embarcada, caminatas en Valle Cretácico, rapel, entre otros) y quienes prestan servicios turísticos con visitas en sus establecimientos ) bodegas u otras actividades gastronómicas y de paseos agroturísticos) (ver tabla 3 ).

Tabla 3 Visitantes y actividades según prestadores de turismo receptivo²

\begin{tabular}{|l|l|c|}
\hline \multicolumn{2}{|c|}{ Prestadores } & 2010-Abril 2011 \\
\hline $\begin{array}{l}\text { Excursiones (Wala Aventuras- Sendas } \\
\text { Patagónicas }\end{array}$ & $\begin{array}{l}\text { Pesca - Flotadas -Margen Sur- Parque } \\
\text { Cretácico - Zoo Bubalcó- Avistaje de aves }\end{array}$ & 730 \\
\hline Zoo - Bubalcó & Actividades en el Zoológico & $\mathbf{1 9 . 0 0 0}$ \\
\hline $\begin{array}{l}\text { Bodegas Agrestis- Establecimiento } \\
\text { de hongos Banquita Laino }\end{array}$ & Actividades de turismo rural y del vino & $\mathbf{1 . 0 1 3}$ \\
\hline Total visitantes & & $\mathbf{2 0 . 7 4 3}$ \\
\hline
\end{tabular}

Fuente: Elaboración propia con datos de los diversos prestadores de turismo

Los prestadores por su parte especialmente en el caso de los que realizan excusiones tienen un ámbito de trabajo extenso, dado por las características propias de la actividad recreativa, y o por la extendida linealidad del DTE Alto Valle. De esta manera se entreteje una red de circuitos que trasciende los municipios y por ende el desarrollo del turismo y la recreación en el Alto Valle.

2 Corresponde a los prestadores que llevan registro de visitas 


\section{CONCLUSIONES}

- La política pública de inclusión del Alto Valle como Destino Turístico Emergente ha resultado positiva para su desarrollo, dado que los programas convergen en grandes objetivos, que promueven sinergia de su crecimiento y de evolución a partir de los diferentes intereses y acciones que surgen del armado de los productos turísticos para la región.

- En una primera etapa, ha movilizado a la recreación de los locales, con un crecimiento muy significativo de demanda recreativa. Esto ha sido y es un factor inicial, de impulso del turismo, ya que los habitantes de la región, en su inmensa mayoría inmigrantes de otras provincias, reciben a familiares, y amigos, los cuales llegan a disfrutar de la oferta Turística que se comercializa. El fuerte desarrollo de la recreación se ha visto posibilitado por la cantidad de habitantes de la región (700.000 aprox.) y su nivel de consumo.

- Los programas en desarrollo combinan planificación e iniciativa que promueven esquemas de trabajo y ciertas etapas de acción con iniciativas locales, donde actores locales son nexo y facilitadores, dejando capacidades instaladas para cada sitio, con microemprendimientos turísticos, Asociaciones civiles, entre otras modalidades. En este sentido, ha sido importante el nivel de comprensión por parte del local, del fenómeno económico - social producido por el desarrollo de las actividades Turísticas, ya que se ha generado un grupo de prestadores de turismo receptivo con desarrollo de eventos, acontecimientos programados con eje en la ruta del vino, excursiones, e inversiones interesantes, entre otras.

- El DTE Alto Valle, posee una economía con fuerte base en la fruticultura para comercio interior y exterior. Esto ha lentificado el ingreso de otra variable de desarrollo como lo es el Turismo- Recreación, ha sido un freno, pero a su vez una oportunidad comercial, dado que un número nada despreciable de empresarios que visitan la zona por negocios, consumen la oferta turística. De esta manera el turismo corporativo de hombre de negocios, contribuye con este nuevo sector económico de la región y son ellos quienes también constituyen una demanda de alto nivel de consumo que ha promovido establecimientos con servicios de alta categoría.

- Finalmente parte de la "fortaleza regional" en el desarrollo del DTE Alto Valle, está dada por la sinergia en las políticas que se han puesto sobre sus hombres todos los 
centros urbanos del Valle, con estructuras destinadas a los servicios de atención al visitante, con diversidad de atractivos que cada administración pone en valor y los privados que se han movilizado por el negocio turístico recreativo, como una forma de vida.

\section{BIBLIOGRAFIA}

Alburquerque, Francisco (1994). "Metodología para el desarrollo económico local". Manual de Desarrollo Local, editado por el Gobierno Vasco (Del Castillo, 1994), Cap. 28. Publicado por REDELALDIA.org (fecha de consulta: 3 abril 2009) Disponible en : http://www.redelaldia.org/IMG/pdf/1114.pdf

Alburquerque, Francisco (2001). La importancia del enfoque del desarrollo económico local. En A. Vázquez Barquero y O. Madoery. (eds.) Transformaciones globales y políticas de desarrollo local. Homo Sapiens ediciones, Rosario, Argentina.

Arocena, José (1995). El desarrollo local: un desafío contemporáneo. Editorial Nueva Sociedad, Caracas.

Boisier, Sergio “Desarrollo Endógeno: ¿Para Qué?, ¿Para Quién? “ El Humanismo en una Interpretación Contemporánea del Desarrollo, Mimeo. Santiago de Chile, 2004

Boscherini, Fabio; Poma, Lucio . Más allá de los distritos industriales: el nuevo concepto de territorio en el marco de la economía global. En Boscherini, Fabio y Poma, Lucio (comp.). En Territorio, conocimiento y competitividad de las empresas. El rol de las instituciones en el espacio global, Miño y Dávila editores, Madrid. 2000

Cotorruelo Menta, Romeo. "Aspectos estratégicos del desarrollo local. En Vázquez Barquero, A. y Madoery, O. (comp.). En Transformaciones globales, Instituciones y Políticas de desarrollo local. Editorial Homo Sapiens, Rosario.2001.http://www.oit.org.ar/portal/programa-cea/index. php?option $=$ com_content $\&$ view $=$ article $\&$ id $=60$ :aspectos-estrategicosdel-desarrollo-local-en-transformaciones-globales-instituciones-ypoliticas-de-desarrollo-local-bg\&catid=11:desarrollo-economicoterritorial\&Itemid=26 (fecha de consulta: mayo 2009)

Elizalde Hevia, Antonio. "Planificación estratégica territorial y políticas públicas para el 
desarrollo local". Instituto Latinoamericano y del Caribe de Planificación Económica y Social (ILPES) - CEPAL - Naciones Unidas. pp 11-25 Santiago de Chile 2003

Garbellotti, Magdalena; González, Myriam; Ñancufil, Adrián, (2009) “Bahía Bustamante: turismo y patrimonio en un pueblo alguero de la Patagonia Argentina" en XII Encuentro de Geógrafos de América Latina. $\quad$ http://observatoriogeograficoamericalatina.org.mx/egal12/ Geografiasocioeconomica/Geografiaturistica/15.pdf (consulta: junio 2011) Madoery, Oscar (2001b). El Proyecto político local como alternativa de desarrollo. En Revista Política y Gestión, volumen 2, UNSAM, Homo Sapiens.

Madoery, Oscar Otro desarrollo: El cambio desde las ciudades y regiones. 1er Edición, San Martín UNSAM EDITA, Universidad Nacional de San Martín, Buenos Aires. 2008

Organización Internacional del Trabajo (OIT). Unidad Didáctica 2 "Desarrollo local y turismo: elementos político-institucionales claves". Centro Internacional de Formación, Programa Delnet de Apoyo al Desarrollo Local, Curso de Turismo Sostenible y Desarrollo Local, Turín. 2009

Otero Urieta, Adriana (2007). La importancia de la visión de territorio para la construcción de desarrollo competitivo de los destinos turísticos. En Cuadernos de Turismo $\mathrm{N}^{\mathrm{o}} 19$, Universidad de Murcia. http://revistas.um.es/turismo/ article/viewFile/13811/13331 (consulta: mayo 2009).

Oyarzun, Edgardo; Szmulewicz, Pablo (1999). Fortalecimientos de la gestión en destinos turísticos. Fundamentos. En Revista Gestión Turística, No 3, Universidad Austral de Chile. http://www.gestionturistica.cl/publicaciones/ revistas/anteriores/4.htm (consulta: julio 2009).

SECTUR - Secretaría de Turismo de la Nación, Argentina (2005). Plan Federal Estratégico de Turismo Sustentable 2016. http://2016.turismo.gov.ar/wp_ turismo/ (Enlace: Institucional, PFETS - Plan Federal). (consulta: mayo 2009)

SECTUR - Secretaría de Turismo de la Nación, Argentina (2009). PROFODE: Programa de Fortalecimiento y Estímulo a Destinos Turísticos emergentes. http://www.turismo.gov.ar/esp/menu.htm (Enlace: Institucional, Desarrollo Turístico, Destinos). (consulta: mayo 2009). 
Toselli, Claudia. (2009) "Programa de fortalecimiento a destinos turísticos emergentes. Un análisis desde la visión del desarrollo endógeno", Gestión Turística $\mathrm{N}^{\circ}$ 12, - 124 ISSN 0717 - 1811, pp109

Turismo Rio Negro (2011). Zonas Turísticas . en sitio web: http://www.rionegrotur.gob.ar/ index.php/zonas-turisticas/zona-valle/atractivos-turisticos/ruta-del-vino.html - consultado en abril 2011.

Vázquez Barquero, Antonio (2001). Desarrollo endógeno y globalización. En Vázquez Barquero, A. y Madoery, O. (comp.). Transformaciones globales, instituciones y políticas de desarrollo local, Homo Sapiens Ed., Rosario.

Vázquez Barquero, Antonio (2000). Desarrollo económico local y descentralización: una aproximación conceptual. CEPAL - GTZ, Santiago de Chile.

Nota: Plan Federal Estratégico de Desarrollo Turístico Sustentable (PFEDTS) entiende por destino emergente aquellos sitios que por sus características naturales y/o culturales presentan potencialidad para atraer turistas tanto nacionales como internacionales, pero que no han alcanzado aún este objetivo debido a problemas de competitividad, o bien a que la oferta turística carece de una puesta en valor adecuada o está desarticulada...."

recibido $24 / 10 / 2011$

aceptado el 11/06/2012

Arbitrado anónimamente. 